\title{
Effects of the Valsalva Maneuver on Intraoperative Bleeding During Tonsillectomy
}

\author{
(D) Ömer Faruk ÇALIM
}

Bezmialem Vakıf University Faculty of Medicine, Department of Otorhinolaryngology, İstanbul, Turkey

\begin{abstract}
Objective: We investigated whether the Valsalva maneuver helps detect any further bleeding points following adequate hemostasis in tonsillectomy.

Methods: This was a prospective study of consecutive patients who underwent tonsillectomy performed in a tertiary medical center. The data collected included age, gender, method of operation, and hemorrhage (if any) informations. Immediately after completing the surgery, hemostasis was performed by an anesthesiologist using the Valsalva maneuver to identify bleeding points. Intraoperative and postoperative hemorrhage, as well as the treatment applied, were recorded.

Results: One hundred twenty patients underwent tonsillectomy (53 males and 67 females). Tonsillectomy was performed using cold dissection in 59 patients ( 26 males and 33 females) and bipolar diathermy in 61 patients ( 27 males and 34 females). The differences in intraoperative hemorrhage following Valsalva were significantly higher in the cold dissection group than in the hot (bipolar) group ( $\mathrm{p}=0.044)$. Tonsillectomy using bipolar cautery had a statistically significantly higher postoperative hemorrhage rate than that using cold dissection $(\mathrm{p}<0.05)$.

Conclusion: The Valsalva maneuver is useful for identifying subtle bleeding vessels in tonsillectomy wound bed. We suggest applying the procedure for final verification of hemostasis before ending the surgery.
\end{abstract}

Keywords: Valsalva maneuver, tonsillectomy, bipolar cautery, cold dissection, hemorrhage

\section{Introduction}

Tonsillectomy is one of the most widespread operations applied in pediatric populations (1). Tonsillectomy has important health advantages, such as avoidance of obstructive breathing and behavioral issues, and improving life quality and neuro-cognition in children (1). Recurrent tonsillitis and/or large tonsil-induced snoring and even obstructive sleep apnea are the most common indications for tonsillectomy (1).

Meticulous hemostasis is important for whole surgical techniques. However, the tonsillar area is especially prone to bleeding because of its rich vascular supply. The tonsil is inclined to bleeding, which might have severe sequels such as bleeding aspiration that could lead to a lethal outcome (2). Different methods have been suggested to optimize intraoperative hemostasis, including the Valsalva maneuver, which may display latent bleeding vessels by increasing the venous pressure. This is particularly important in tonsillectomy practice where surgical drains cannot be used.

Tonsillectomy complications include intraoperative and postoperative hemorrhage, respiratory complications, nausea, vomiting, pain, and burn injuries. Post-tonsillectomy hemorrhage, which occurs in $2.7-5 \%$ of children (3), can be a life-threatening complication that requires emergent surgery. Furthermore, risks of anesthesia- and opioid-related respiratory depression 
are other mortal complications that may occur during or after tonsillectomy. Control of post-tonsillectomy hemorrhage usually can be performed easily; however, in a few patients, this may be a challenge (4).

The Valsalva maneuver is performed by forceful attempted exhalation against a closed glottis.

During the maneuver, the intra-thoracic pressure becomes extremely positive and impedes venous return into the thorax. These changes increase the peripheral venous pressure. In our study, the Valsalva maneuver was performed intra-operatively by the attending anesthetist.

We assessed the effectiveness of the Valsalva maneuver for preventing bleeding in the tonsillar wound bed intra-operatively and postoperatively following cold dissection and bipolar diathermy. In addition, we determined whether there was any difference in the intraoperative and postoperative hemorrhage rates between the two methods.

\section{Methods}

A prospective, self-controlled clinical study was conducted to confront the influences of cold towards bipolar electro cautery tonsillectomy procedures in patients with recurrent chronic tonsillitis. One hundred twenty patients aged below 16 years, who were admitted for tonsillectomy were enrolled. All procedures performed in studies involving human participants were in accordance with the ethical standards of the institutional and/or national research committee and with the 1964 Helsinki Declaration and its later amendments or comparable ethical standards. This study protocol was confirmed by the Bezmialem Vakıf University Clinical Research Ethics Committee (18.04.2017-8/79). Informed consent was taken from all individual subscribers including the parents of the patients. Patients underwent tonsillar biopsy, tonsillectomy with palatal surgery, pediatric patients with known hematological abnormalities or hypertension and patients in whom general anesthesia was contraindicated were excluded. Before surgery, antibiotic and dexamethasone $(0.15 \mathrm{mg} / \mathrm{kg})$ treatments were given parenterally to all patients. Surgery was applied under general anesthesia. Patients were grouped according to the methods of dissection and hemostasis. In the first method, bipolar diathermy was used for both dissection and hemostasis. Both tonsils were dissected together with their capsules and the tonsils were removed using a bipolar electro cautery instrument (ME 411; KLS Martin, Tuttlingen, Germany) set in the cutting mode by power of 20-35 W. The second method involved a cold dissection technique (scissors and raspatory) with hemostasis using packs; however, if bleeding continued, diathermy was used for hemostasis. After the completion of tonsillectomy and performing hemostasis using a bipolar diathermy technique, the Valsalva maneuver was performed, at least twice, for $30 \mathrm{~s}$ by applying $30 \mathrm{~cm}$ of positive end-expiratory pressure to the ventilator circuit. Meanwhile, bleeding points were identified and treated using bipolar diathermy. Complications included early bleeding within $24 \mathrm{~h}$ and late postoperative bleeding causing readmission for surveillance, return to the operation room, or blood transfusion. In addition, on the postoperative first day, the patients were examined to reveal any bleeding before hospital discharge.

All of the patients were hospitalized for one day in the postoperative period. Peroral analgesics and antibiotics were given during 10 days, postoperatively. After discharge, the patients were demanded to return to the hospital if hemorrhage emerged. The patients were examined after 10 days. Patients or their relatives were asked whether any postoperative bleeding had occurred within 10 days after discharge.

\section{Statistical Analysis}

All of the statistical analyses were made using the SPSS 12.0. Descriptive statistics (median, standard deviation, mean, minimum and maximum rates) and frequencies were calculated for numerical and categorical variables, respectively. The Mann-Whitney $U$ test was used to confront intraoperative and postoperative hemorrhage between two methods. Variance analysis was used to evaluate the relationship between hemorrhage and age. A significance level of $p$ less than 0.05 was accepted.

\section{Results}

One hundred twenty patients underwent tonsillectomy during the study period (53 males and 67 females; mean age, 9.4 years). Tonsillectomy was performed using cold dissection in 59 patients (26 males and 33 females; mean age, $9.5 \pm 5.2$ years) and bipolar diathermy in 61 patients ( 34 females and 27 males; mean age, $8.9 \pm 5.4$ years).

The number of females was higher than males, without reaching a statistical significance $(\mathrm{p}=0.70)$. No significant difference was considered between the two groups in terms of age and early hemorrhage (first day of surgery). The differences in intraoperative hemorrhage following Valsalva were significantly higher in the cold dissection group than in the hot (bipolar) group $(\mathrm{p}=0.044$; Table 1, Figure 1). Tonsillectomy using bipolar cautery had a statistically significantly higher postoperative hemorrhage rate than that using cold dissection ( $\mathrm{p}<0.05$; Figure 2$)$.

\section{Discussion}

Previous studies have shown that, after tonsillectomy, postoperative hemorrhage is higher when using hot dissection (monopolar, bipolar, or coblation) than using cold dissection, and that there is a dose-response relationship between postoperative hemorrhage and the bipolar diathermy power setting (5). The Valsalva maneuver has been used to check bleeding points after hemostasis in thyroidectomy procedures (6); however, a review of the literature revealed no information

\begin{tabular}{|c|c|c|}
\hline & $\begin{array}{l}\text { Intraoperative } \\
\text { bleeding }\end{array}$ & $\begin{array}{l}\text { Postoperative } \\
\text { bleeding (10 days) }\end{array}$ \\
\hline Cold dissection group (n) & 9 & 2 \\
\hline Hot dissection group (n) & 3 & 7 \\
\hline
\end{tabular}




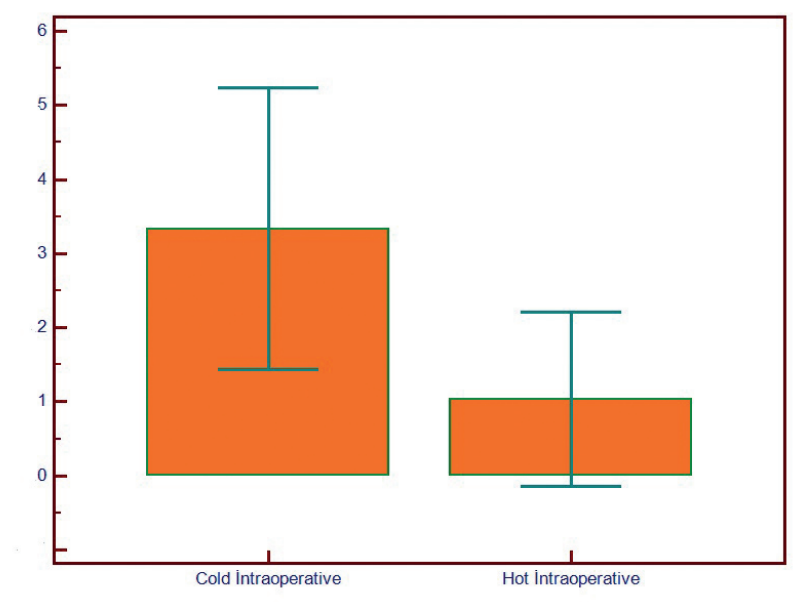

Figure 1. After the Valsalva maneuver comparison between two groups in terms of intraoperative haemorrhage rate

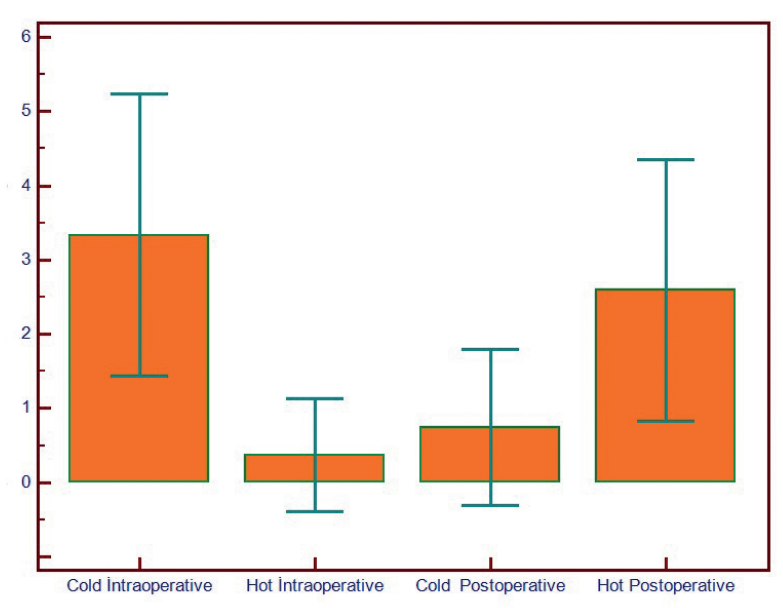

Figure 2. The comparison of intraoperative and postoperative haemorrhage rates

concerning the relationship between the Valsalva maneuver and the threat of post-tonsillectomy bleeding. In the present study, after adequate hemostasis, we performed the Valsalva maneuver intra-operatively and compared the risk of hemorrhage between cold and hot dissection tonsillectomy. We found that the risk of intraoperative hemorrhage was statistically significantly higher in the cold dissection group than in the bipolar diathermy group. Conversely, the risk of postoperative hemorrhage was higher in the bipolar diathermy group. The cold dissection group was subjected to the cold dissection technique (scissors and raspatory) with hemostasis using packs; and if bleeding continued, diathermy was used for hemostasis. After hemostasis, the Valsalva maneuver was performed.

Tonsillectomy dissection is commonly applied by cold or diathermy dissections. Hemostasis may be achieved using diathermy, ligature bonds to bleeding vessels, or both of them. Diathermy devices may be unipolar or bipolar. While the reported post-tonsillectomy hemorrhage rates vary with the effort of postoperative examination, the published rates for primary hemorrhage during the first $24 \mathrm{~h}$ after tonsillectomy vary from $0.3 \%$ to $2.1 \%$, and secondary bleeding rates necessitating at least incoming to the hospital vary from $2 \%$ to $10.3 \%(7)$. Many publications have defined (8) varied ratios of postoperative hemorrhage in "cold" tonsillectomy procedures and "hot" tonsillectomy procedures, usually identifying lower bleeding rates after cold techniques. In the present study, postoperative hemorrhage was significantly higher in the hot (bipolar) group than in the cold dissection group.

The differences among articles regarding bleeding rates after tonsillectomy using varied procedures need to be discussed. Higher post-tonsillectomy bleeding rates following diathermy techniques may be related to more thermal damage because of extremely high power settings or more frequent or prevalent application of diathermy (5).

The United Kingdom National Prospective Tonsillectomy Audit (NPTA) announced its final survey of tonsillectomies in 2005 (8). According to the NPTA report, cold dissection plus hemostasis using bonds or packs had the lowest risk of posttonsillectomy bleeding. If the odds ratio for post-tonsillectomy bleeding using this technique is set at 1 , then cold dissection plus hemostasis using unipolar or bipolar diathermy has a 1.6 times greater risk for hemorrhage (adjusted odds ratio). Unipolar cautery or bipolar diathermy dissection and hemostasis showed a 2.5- to 3.2-times greater risk for bleeding. All of these differences reached statistical significance. Only diathermy method had a lower risk for primary bleeding than that techniques (9). This present study aimed to reduce intraoperative and postoperative bleeding by performing the Valsalva maneuver. However, similar to other studies reported in the literature, intraoperative hemorrhage was significantly higher in the cold dissection group than in the hot (bipolar) group.

\section{Study Limitations}

Low number of participants and the lack of comparison of other techniques used in tonsillectomy are among the limitations of the study. However, our study is a preliminary study for future research.

\section{Conclusion}

An obvious difference in intraoperative bleeding was observed between the two methods in response to the Valsalva maneuver. Our results show that the Valsalva maneuver is useful for identifying bleeding vessels for thorough tonsillectomy homeostasis. The procedure may become a helpful addition for final verification of hemostasis before ending surgery.

\section{Ethics}

Ethics Committee Approval: This study protocol was confirmed by the Bezmialem Vakıf University Clinical Research Ethics Committee (18.04.2017-8/79).

Informed Consent: Informed consent was taken from all individual subscribers including the parents of the patients. 
Peer-review: Internally peer-reviewed.

Financial Disclosure: The author declared that this study received no financial support.

\section{References}

1. Subramanyam R, Varughese A, Willging JP, Sadhasivam S. Future of pediatric tonsillectomy and perioperative outcomes. Int J Pediatr Otorhinolaryngol 2013;77:194-9.

2. Windfuhr JP, Schloendorff G, Baburi D, Kremer B. Serious posttonsillectomy hemorrhage with and without lethal outcome in children and adolescents. Int J Pediatr Otorhinolaryngol 2008;72:1029-40.

3. Venkatesan NN, Rodman RE, Mukerji SS. Post-tonsillectomy hemorrhage in children with hematological abnormalities. Int J Pediatr Otorhinolaryngol 2013;77:959-63.
4. Blakley BW. Post-tonsillectomy bleeding: how much is too much? Otolaryngol Head Neck Surg 2009; 140:288-90.

5. Lowe D, Cromwell DA, Lewsey JD, Copley LP, Brown P, Yung M, et al. Diathermy power settings as a risk factor for hemorrhage after tonsillectomy. Otolaryngol Head Neck Surg 2009;140:23-8.

6. Moumoulidis I, Martinez Del Pero M, Brennan L, Jani P. Haemostasis in head and neck surgical procedures: Valsalva manoeuvre versus Trendelenburg tilt. Ann R Coll Surg Engl 2010;92:292-4.

7. O'Leary S, Vorrath J. Postoperative bleeding after diathermy and dissection tonsillectomy. Laryngoscope 2005;115:591-4.

8. Lowe D, van der Meulen J. National Prospective Tonsillectomy Audit. Tonsillectomy technique as a risk factor for postoperative haemorrhage. Lancet 2004;364:697-702.

9. Blanchford H, Lowe D. Cold versus hot tonsillectomy: state of the art and recommendations. ORL J Otorhinolaryngol Relat Spec 2013;75:136-41. 